\title{
The Master-Curve Band considering Measurement and Modeling Uncertainty for Bituminous Materials
}

\author{
Quan Liu $\left(\mathbb{D},{ }^{1}\right.$ Jiantao Wu $\left(\mathbb{D},{ }^{2}\right.$ Pengfei Zhou $\mathbb{D},{ }^{2}$ and Markus Oeser $\mathbb{D}^{1}$ \\ ${ }^{1}$ Institute of Highway Engineering, RWTH Aachen University, Aachen 52074, Germany \\ ${ }^{2}$ College of Civil and Transportation Engineering, Hohai University, Nanjing 210037, China \\ Correspondence should be addressed to Jiantao Wu; jiantao.wu@hhu.edu.cn
}

Received 24 January 2021; Revised 13 February 2021; Accepted 19 February 2021; Published 25 February 2021

Academic Editor: Shuaicheng Guo

Copyright (C) 2021 Quan Liu et al. This is an open access article distributed under the Creative Commons Attribution License, which permits unrestricted use, distribution, and reproduction in any medium, provided the original work is properly cited.

\begin{abstract}
This paper proposes using the master-curve band (MCB) to incorporate the unavoidable measurement errors and modeling uncertainty into the bitumen master-curve construction. In general, the rheological property of bitumen within the linear viscoelastic region is characterized by the master curve of modulus and/or phase angle, provided that the bitumen complies with the time-temperature superposition principle (TTSP). However, the master-curve construction is essentially a mathematical fitting process regardless of whether or not the original data is perfect enough to fit. For this reason, the MCB was introduced to consider the uncertainty information instead of a single master curve. Rheological data of four kinds of bitumen including unaged and aged bitumen were used to construct the MCBs. The results indicated that the generalized sigmoidal model showed the widest master-curve band, followed by Christensen-Anderson-Marasteanu (CAM) and CAM $\left(G_{g}\right)$ models. The width of MCB was a useful tool to identify the sensitivity of bitumen to rheological models. The sensitivity of bitumen to rheological models is associated with the number of active parameters in rheological models and model parameters' confidence intervals. The construction of an MCB was beneficial to select the rheological models. Accordingly, the CAM $\left(G_{g}\right)$ model is proved to be the best to analyze the aging effects.
\end{abstract}

\section{Introduction}

Most of the distresses that occur in asphaltic pavements are associated with the binder phase in asphalt mixtures, such as adhesion failure (moisture damage) [1, 2], cohesion failure within bitumen (cracking) [3], fatigue failure [4, 5], and permanent deformation (rutting) [6]. In the characterization of bitumen's physical properties, rheological behavior was stressed due to its close connection to the above distresses [7]. The terminology rheology was conceptualized as the study of deformation and flow properties of materials [8]. Bituminous material is manifested as being viscoelastic, showing typical time- and temperature-dependent properties [9]. By far, the rheological properties of bitumen are conventionally represented by two primary parameters, which are complex shear modulus $\left(G^{*}\right)$ and phase angle $(\delta)$ measured through the dynamic shear rheometer (DSR).
It is worth noting that the complex shear modulus and phase angle of bitumen only make sense with a given temperature and loading frequency. However, the property of bitumen under a single condition of temperature and loading frequency is not sufficient to reveal the entire performance of bitumen in a broad range of temperature and frequency. For this reason, Airey [8] proposed the black diagram to identify the rheological performance without considering the temperature and frequency. To date, the black diagram has been successfully used in many studies of bitumen rheology $[7,10,11]$. However, the black diagram is not able to reveal the temperature or frequency sensitivity of bitumen. Alternatively, the master curve of modulus or phase angle was proposed to handle the rheological data [12-14]. The master-curve construction introduced a time-temperature shift factor to bring rheological data measured at different temperatures and frequencies to one overall continuous curve. The preparatory 
work required for constructing the master curve is to determine the shift factor as a temperature function. Many approaches were proposed to determine the temperature dependence of shift factors [15], such as a manual procedure [16], the Arrhenius equation [17], the Williams-Landel-Ferry (WLF) formula [18], logarithmic-linear relationship with temperature [19], the Fox method [20], and viscosity-temperature susceptibility (VTS) method [21].

In addition to the determination of shift factors, rheological models are usually used to achieve a perfectly smooth master curve. Yusoff et al. [22] classified rheological models into three categories: nonlinear multivariable models, empirical algebraic equations, and mechanical element models. The nonlinear multivariable models are not usually used as they are empirical models presented in the form of a figure. The empirical algebraic equations, such as the sigmoidal model and Christensen-Anderson-Marasteanu (CAM) model, and mechanical element models, such as the 2S2P1D model, were extensively adopted in many kinds of research. The measured rheological data is mathematically fitted through an optimization process, converting into the master curve at the reference temperature. Herein, it is noticeable that the achieved mater curve, to some extent, mathematically approximates the experimental data. However, uncertainty in the above process could cause significant variation between experimental and predicted data. Figure 1 illustrates the potential uncertainty factors that could give rise to variation between measurement and prediction.

Among these potential uncertainty factors shown in Figure 1, sample variation can be eliminated by measuring multiple parallel samples. As for the testing protocols, some efforts have been made toward accurate measurement for decades. Carswell et al. [23] discussed testing protocols that might cause measurement variation: temperature control, sample geometry, sample preparation, equipment calibration, linear range, and so forth. In their discussion, temperature control and sample geometry are two critical sources of errors. With the development of the device and correlation method, temperature control is no longer an issue if the standard procedure is followed. The geometry size effect can be eliminated by using appropriate testing plates [8]. The bitumen sample showed high modulus in some conditions, and the compliance error could induce inaccurate measurement. Correspondingly, Wu et al. [24] proposed an efficient method to correct rheological data's compliance error using the 2S2P1D model.

Nonetheless, the measurement uncertainty caused by laboratory or technician difference cannot be avoided (referring to measurement error in Figure 1). A study launched by RILEM investigated the repeatability of binder fatigue tests [25]. This study invited varying laboratories to measure the same type of bituminous materials. In this report, the coefficient of variation measured for complex modulus was up to $15.6 \%$. It is illustrated that the measurement error of rheological properties is practically unavoidable.

On the other hand, in the practical realization of the master curve, the determination of shift factors is flexible toward a goodness of fit of rheological models. Therefore, the variation of shift factors usually depends on the rheological model selection. In this case, unavoidable uncertainty mainly rests on the measurement error, model variation, and fitting variation.

Based on the above analysis, finding an approach that incorporates uncertainty information into the master curve could benefit a more accurate estimation of bitumen rheological properties. For this reason, this study proposed a method to consider the uncertainty that exists in mastercurve construction by extending a master curve to a mastercurve band (MCB). The MCB has the following potential advantages compared with the master curve: (1) the uncertainty information is included in the master-curve band; (2) the sensitivity of bitumen to the rheological model can be quantitatively featured; and (3) the MCB can help select appropriate rheological models to study the aging or modification effect.

\section{The Development of the Master-Curve Band}

2.1. Data Variation Measured by DSR. In the first phase of the RILEM Round Robin Binder Fatigue Test mentioned above, it was found that the coefficient of variation (COV) measured for complex modulus was $15.6 \%$, and, for phase angle, the maximum coefficient of variation observed was $3.7 \%$ [25]. After improving the test protocols, in the second phase of their study, the variation coefficient changed to $6.7 \%$ and $4.8 \%$ for complex modulus and phase angle, respectively [25]. Although this study was carried out to identify reasonable fatigue test methods, the results indicated that DSR measurement showed unavoidable measurement errors for complex modulus and phase angle. Besides, the measurement error of the complex modulus is much more significant than that of the phase angle. Unfortunately, a single master-curve cannot include the data variation in its current presentation form.

\subsection{Model Variation Caused by Model Types and Fitting} Process. Many linear viscoelastic rheological models have been developed to construct the master curve of bitumen. Empirical equations such as CAM and mechanical models such as the 2S2P1D model have been successfully applied to characterize the bitumen viscoelastic properties. Nur et al. compared several mathematical models applied to the unaged, aged, and modified bitumen binders [26]. The results showed that the generalized sigmoidal model performed the best in terms of the goodness of fit, while the CAM model was reported to be senior to other models. Nonetheless, modeling sensitivity has not been thoroughly investigated. One rheological model might be powerful enough to satisfy measurement data. However, it cannot exclude the possibility of overfitting. Therefore, a good quality model should show the goodness of fit and efficiently identify the variation caused by data variation and modeling uncertainty.

2.3. Quantitative Description of Data Variation and Modeling Uncertainty. This section introduced how to consider the 


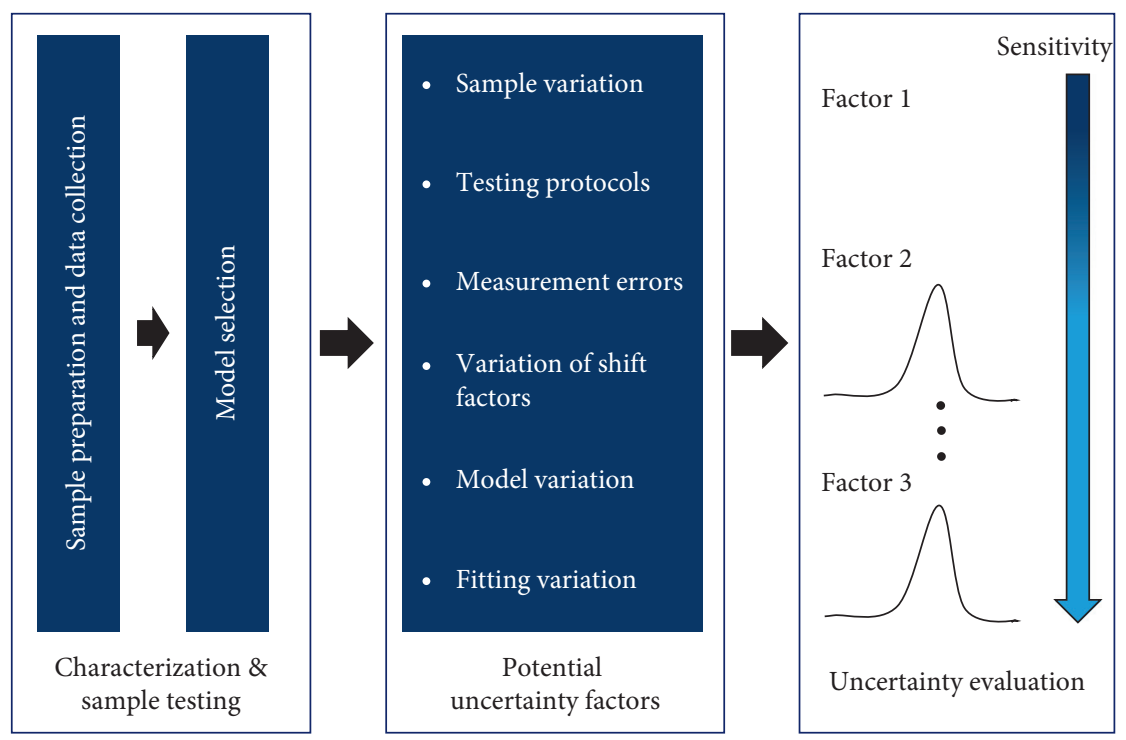

Figure 1: Qualitative analysis of uncertainty factors in master-curve construction.

data variation and modeling uncertainty quantitatively. As previously noted, the unavoidable data variation could reach up to $15.6 \%$. Therefore, the measured data was extended by multiple corresponding coefficients of variation, as shown in Figure 2(a). Seven data groups, including the measured one, were calculated.

Seven data groups were then subjected to the construction of a master curve using a specific rheological model. In this case, seven groups of model parameters can be obtained. It is time-consuming to conclude the distribution of model parameters experimentally. Alternatively, in previous literature, it was reported that measurements of asphalt mixtures reasonably approximate a normal distribution curve [27]. Therefore, it was assumed that the distribution of model parameters caused by modeling uncertainty also complies with the normal distribution, as shown in Figure 2(b).

Moreover, this study considered three confidence levels $(95 \%, 90 \%$, and $85 \%)$ to study the model sensitivity of bitumen. It should be stressed that this study only focuses on the development of a master-curve band for complex modulus. However, the master-curve band's construction for phase angle can also be achieved following the identical procedure proposed in this study.

2.4. The Mathematical Expression of a Master-Curve Band. For example, the rheological model, namely, the generalized sigmoidal model and the Williams-Landel-Ferry (WLF) formula, was used to construct the master curve. The WLF equation is as follows [15]:

$$
\log \left(\alpha_{T}\right)=\frac{-C_{1}\left(T-T_{\text {ref }}\right)}{C_{2}+\left(T-T_{\text {ref }}\right)}
$$

where $C_{1}$ and $C_{2}$ are two constants relative to material properties.
The generalized sigmoidal model was proposed to describe the stiffness of asphalt mixtures. It has been proven applicable to the bituminous binder and other materials, as shown in the following equation [28]:

$$
\log \left|G^{*}\right|=\delta+\frac{\alpha}{\left[1+\lambda e^{(\beta+\gamma\{\log (2 \pi f)\})}\right]^{1 / \lambda}},
$$

where $G^{*}$ is the complex modulus and $f$ is the reduced frequency. $\delta, \alpha, \beta, \lambda$, and $\gamma$ are model parameters.

According to the time-temperature superposition principle, the complex modulus measured at temperature $T$ and frequency $f$ can be obtained at reference temperature $T_{r e f}$. The reduced frequency is determined through the shift factor, as expressed in the following equation [29]:

$$
G^{*}\left(T, \alpha_{T} \cdot f\right)=G^{*}\left(T_{\text {ref }}, f\right) .
$$

The construction of the master curve was performed with the aid of the Solver function in Excel. Combining equation 1 with equation 3 , the master curve can be derived by optimizing the parameters with nonlinear least-square regression methods. In particular, this method minimized the sum of square errors (SSE) between measured data and predicted data, which can be written as follows [30]:

$$
\operatorname{SSE}=\sum \frac{\left(\log \left|G_{\text {mea }}^{*}(f, T)\right|-\log \left|G_{\text {mod }}^{*}\left(\alpha_{T}\left(T, T_{\text {ref }}\right) \cdot f, T_{\text {ref }}\right)\right|\right)^{2}}{\left(\log \left|G_{\text {mea }}^{*}(f, T)\right|\right)^{2}} .
$$

The model parameters were achieved by solving equation 4 , consisting of a parameter vector $\boldsymbol{x}=\left(x_{1}, x_{2}, \cdots, x_{n}\right)$. It is hypothesized that individual parameters are independent of each other. As introduced previously, each parameter should not be a specific value but a range, as shown in equation 5 . The normal distribution fitting can determine the lower and upper values for a single parameter. 


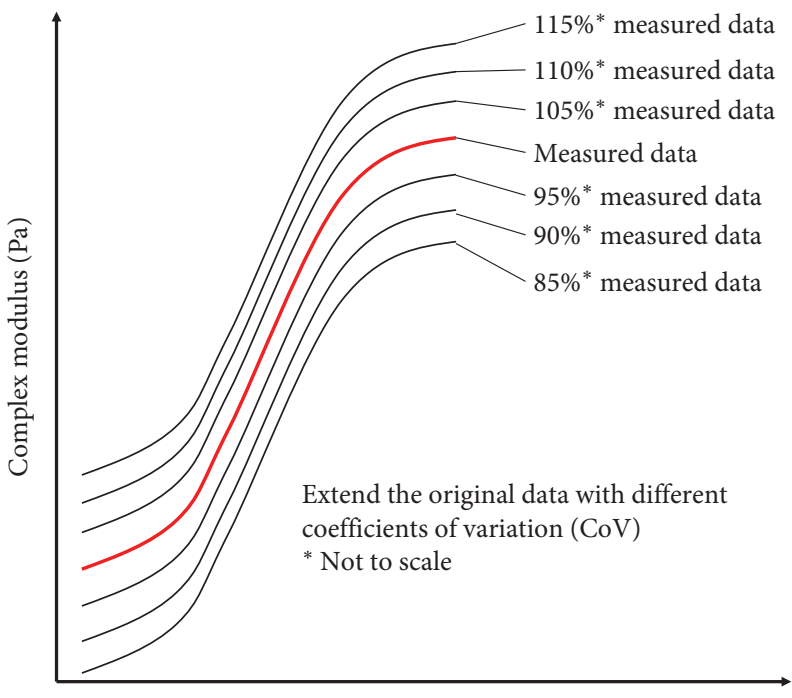

Log reduced frequency $(\mathrm{Hz})$

(a)

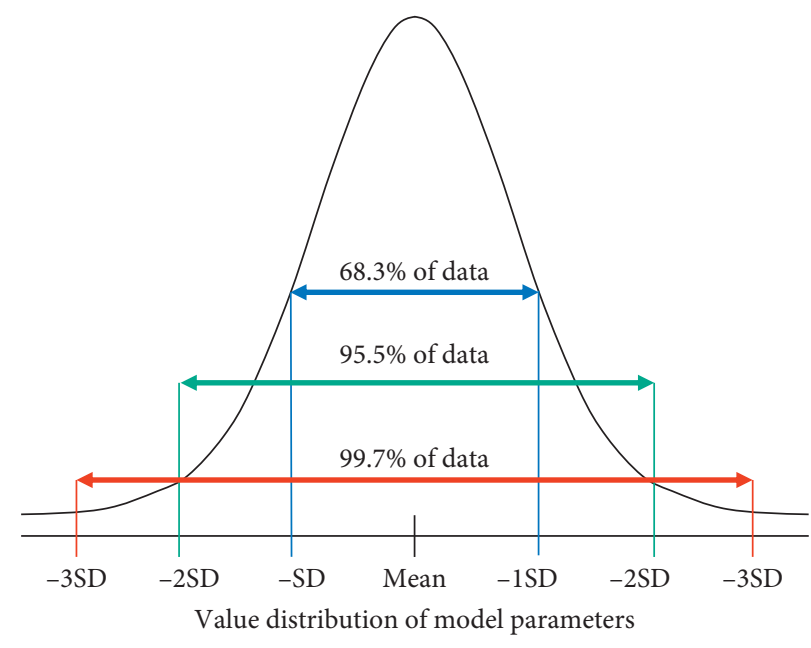

(b)

Figure 2: Qualify the data variation and modeling uncertainty quantitatively. (a) Data variation. (b) Modeling uncertainty.

$$
X_{i}=\left[x_{i}^{l}, x_{i}^{u}\right]
$$

Based on equation 5 , the parameter vector $x$ can be extended to data space $U$, as expressed in the following equation:

$$
U=\left\{X \mid x_{i} \varepsilon\left[x_{i}^{l}, x_{i}^{u}\right], \quad i=1,2,3,4, \ldots, n\right\} .
$$

Accordingly, the single master curve is extended to a master-curve band. Using a generalized sigmoidal model, the master-curve band can be induced as the two following equations:

$$
\begin{gathered}
M C B=\left\{S \mid S_{i}=\delta_{i}+\frac{\alpha_{i}}{\left[1+\lambda_{i} e^{\left(\beta_{i}+\gamma_{i}\{\log (2 \pi f)\}\right)}\right]^{\left(1 / \lambda_{i}\right)}}, \quad i=1,2,3,4, \ldots, \infty\right\}, \\
\left(\alpha_{i}, \beta_{i}, \delta_{i}, \gamma_{i}, \lambda_{i}\right) \in U, \quad i=1,2,3,4, \ldots, \infty .
\end{gathered}
$$

2.5. Procedure to Construct the Master-Curve Band. This section explained how to draw a master-curve band step by step, as shown in Figure 3. After the rheological test, reference temperature was firstly determined. In what follows, the shift factors are determined based on the WLF or Arrhenius equations. The rheological model's selection is subjective, which could be any empirical or mechanical models. Once the rheological model is determined, model parameters can be obtained by solving the target minimization function. The measured isochronal plots are multiplied by the coefficients from $85 \%$ to $115 \%$ for the master-curve band construction. In total, six other groups of isochronal plots are calculated.

The normal distribution fit is then performed separately at $85 \%, 90 \%$, and $95 \%$ confidence levels. The data space $U$ in equation 6 for each parameter is obtained at a specific confidence level. According to equation 7, the master-curve band is generated by performing a calculating experiment using MATLAB code. All possible values in data space $U$ are considered. Figure 4 illustrates an example of extending from master curve to master-curve band using a generalized sigmoidal model.

2.6. Data Collection and Selected Rheological Models. Four kinds of bitumen were subjected to DSR measurements to obtain rheological data. Two of them were unaged and unmodified bitumen, marked as U15 and U70. The numbers following " $U$ " indicated the penetration grade. One bitumen was the aged U15, noted as A15. The other one was the SBS modified bitumen based on U70, coded as SBS. All the samples were subjected to the DSR frequency sweep following the standard procedure.

Two rheological models were used to construct the master-curve band: generalized sigmoidal model 


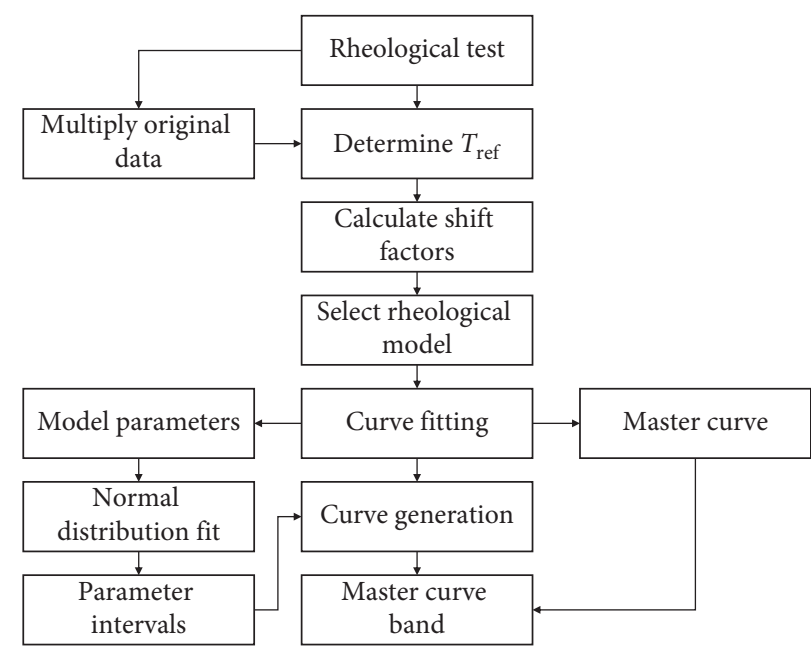

FIgURE 3: Schematic of master-curve band construction.

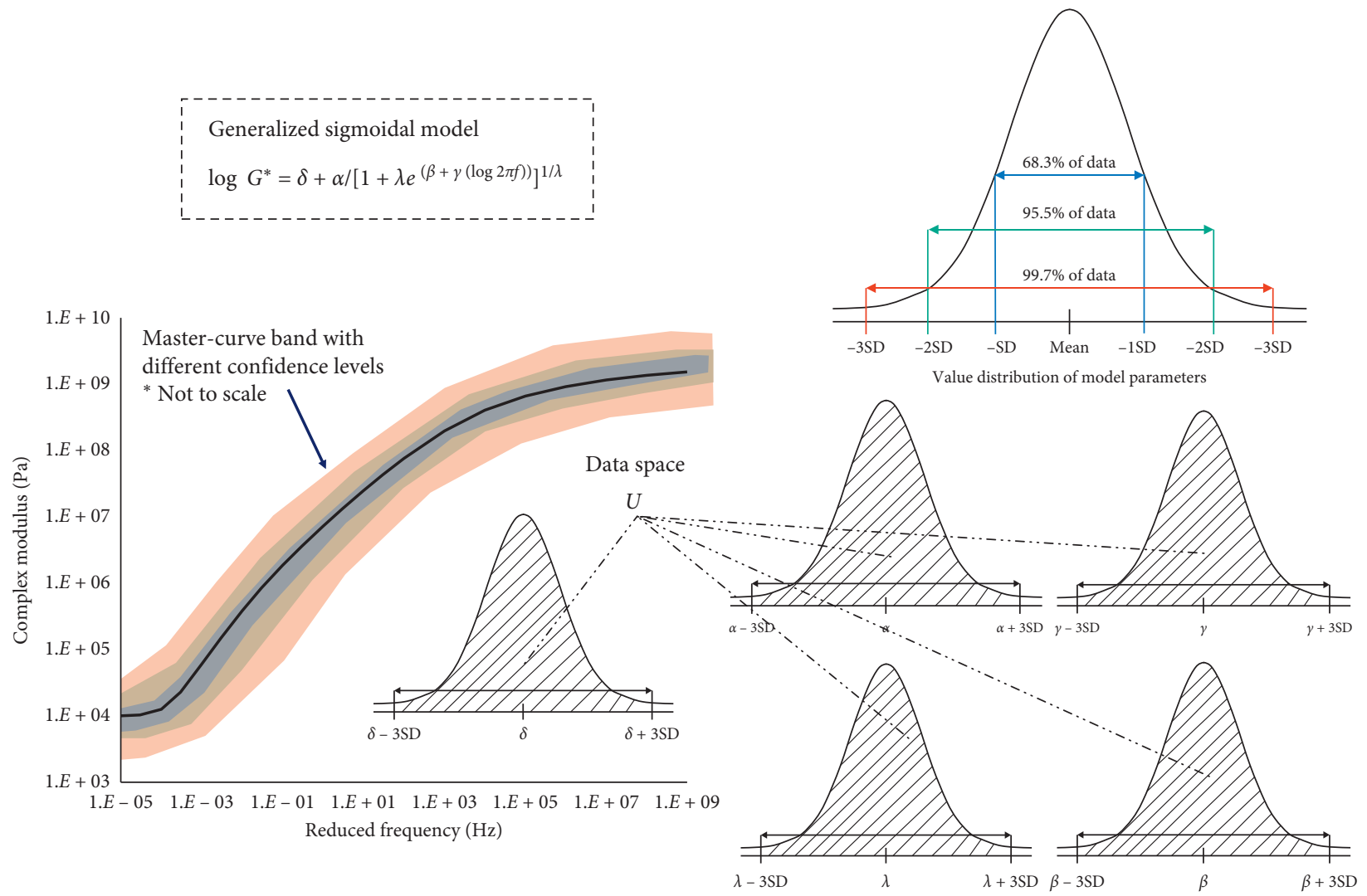

FIgURE 4: Extension from master curve to MCBs (example: generalized sigmoidal model).

(introduced above, GS) and CAM model. According to a previous study, bitumen's theoretical glassy modulus is supposed to be $1 \mathrm{GPa}$ (shear test). Therefore, the CAM model was utilized in two ways. One refers to the CAM model with the glassy modulus fixed at $1 \mathrm{GPa}$. The other one with the glassy modulus unfixed was noted as CAM $\left(G_{g}\right)$ model.
The CAM model is mathematically represented as follows [31]:

$$
\left|G^{*}\right|=G_{g}\left[1+\left(\frac{\omega_{c}}{\omega}\right)^{v}\right]^{-(w / v)}
$$

where $G_{g}$ represents the glassy modulus, $\omega_{c}$ is the crossover frequency, and $v$ and $w$ are model parameters. 


\section{Basic Properties of the Master-Curve Band}

The master-curve band is conceptualized by incorporating the measurement and/or modeling uncertainty into the master curve. However, very few previous studies introduced the master-curve band as well as the basic properties of the master-curve band. Besides, the rheological model's effect on the master-curve band is still unknown. The basic properties varied with different confidence levels and the bitumen types are vague. Therefore, this section discusses the basic properties of the master-curve band regarding the effect of rheological models, bitumen types, confidence intervals, and bitumen aging, respectively.

3.1. Effect of Rheological Models. Figure 5 presents the master-curve bands of bitumen U15 constructed using three different models at a 95\% confidence level. It can be seen that, despite using the same data, different models can result in different master-curve bands. The generalized sigmoidal model showed the widest band, followed by the CAM and CAM $\left(G_{g}\right)$ models. It indicates that the CAM $\left(G_{g}\right)$ model performed the best in modeling $\mathrm{U} 15$ as the least deviation caused by modeling. The only difference between CAM and CAM $\left(G_{g}\right)$ models rests on whether the glassy modulus was fixed. However, the CAM model, which has one parameter less than the CAM $\left(G_{q}\right)$ model, showed a wider band. It indicates that the fix of the glassy modulus would change the values of other parameters. In this sense, the CAM model and CAM $\left(G_{g}\right)$ model are essentially two different models. Table 1 presents the parameter intervals calculated at a confidence level of $95 \%$. It can be seen that the GS model owns five model parameters, which contributed to its wider master-curve band. Although the CAM model has only three model parameters, the interval of $w_{c}$ was from 17.61 to 28.32, while in the CAM $\left(G_{g}\right)$ model the value was only from 1.23 to 1.42 . Therefore, it is not true that the band width solely depends on the number of model parameters. Parameter interval is another critical factor that influences the width of the master-curve band.

On the other hand, it can be concluded that $v$ and $w$ are two model parameters that will not significantly change due to data variation. In this study, parameters with such kind of character are called inert parameters. In contrast, parameters with a considerable change due to data variation are called active parameters. The inert and active parameters can be distinguished by calculating the variation coefficient with a cut-off value of $20 \%$. In this case, the numbers of active parameters in the GS, CAM $\left(G_{g}\right)$, and CAM models are four, two, and one, respectively.

3.2. Effect of Bitumen Types. Figure 6 shows the mastercurve bands of different bitumen binders using the GS model at a 95\% confidence level. Various bitumen binders responded differently to the identical construction procedure of master-curve bands. As shown in Figure 6, the MCB of U70 showed the widest band, followed by U15 and SBS. The band width reflects the model sensitivity of bitumen. Herein, modified bitumen investigated in this study

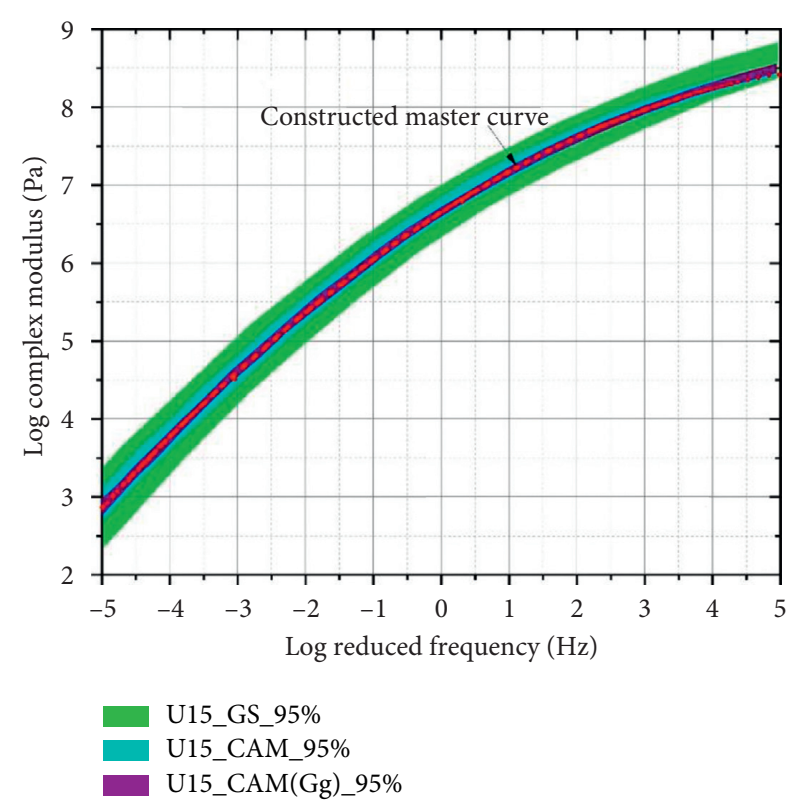

FIGURE 5: Master-curve bands constructed using different models.

showed the smallest sensitivity to the data variation. Provided that an identical measurement variation occurred, the master curve of modified bitumen could present the smallest deviation to the correct master curve. Similarly, hard and unmodified bitumen showed less deviation compared to softer and unmodified bitumen in this study.

To further explain the sensitivity of bitumen to rheological models, the confidence intervals for different bitumen are presented in Table 2. For U15, as the confidence level increased, the interval was enlarged except for the parameter $\gamma$. It indicated that the parameter $\gamma$ is relatively stable when using the GS model to construct U15 even in the presence of data variation. However, for other parameters, data variation could result in a change of parameter value.

In terms of U70, the model parameters can be divided into two groups. The first group (active group), including $\delta$, $\alpha$, and $\lambda$, complied with the tendency found in U15. The other group (including $\beta$ and $\gamma$ ) is the inert group, in which the interval remained almost unchanged as the confidence level increased. In this case, two model parameters would not be significantly altered due to the data variation.

Nevertheless, the variation of U70 between the lower and upper values is relatively more considerable than that of $\mathrm{U} 15$, which is ascribed to a wider master-curve band. In the case of SBS, the parameter $\lambda$ was also classified into the inert group. However, the parameter interval in the active group was the smallest compared to that of U70 or U15.

It can be concluded that the model sensitivity of bitumen is correlated with not only the type of rheological model but also the type of bitumen.

3.3. Effect of Confidence Levels. Figure 7 presents the MCBs of U15 constructed at different confidence levels. When the rheological model was the GS model, the increase of confidence level increased MCB width. As the confidence level 
TABLE 1: Estimated parameter intervals of U15 at confidence level 95\%.

\begin{tabular}{cccccccccccccc}
\hline & \multicolumn{4}{c}{} & \multicolumn{3}{c}{ GS model } & \multicolumn{4}{c}{ CAM $\left(G_{g}\right)$ model } & \multicolumn{4}{c}{ CAM model } \\
& $\delta$ & $\alpha$ & $\lambda$ & $\beta$ & $\gamma$ & $G_{g}(\mathrm{GPa})$ & $w_{c}$ & $v$ & $w$ & $w_{c}$ & $v$ & $w$ \\
\hline Lower & 10.09 & -11.37 & 3.52 & 2.18 & 0.57 & 1.95 & 1.23 & 0.12 & 1.22 & 17.61 & 0.15 \\
Upper & 10.32 & -10.59 & 3.66 & 2.46 & 0.58 & 2.46 & 1.42 & 0.12 & 1.23 & 28.32 & 0.16 & 1.05 \\
\hline
\end{tabular}

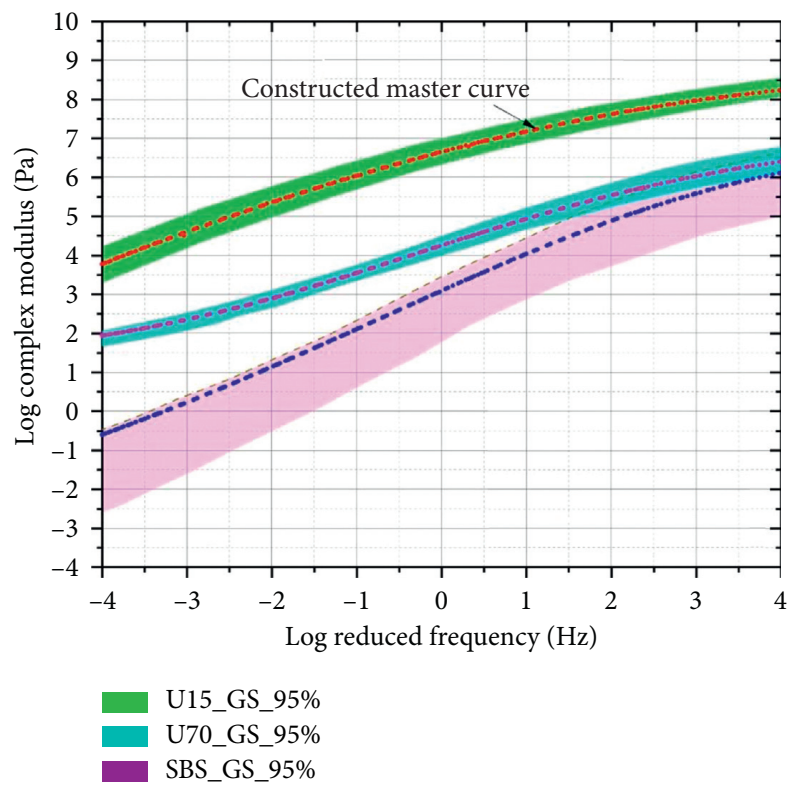

Figure 6: Master-curve bands of different bitumen binders.

TABle 2: The confidence interval constructed for different bitumen using the GS model.

\begin{tabular}{|c|c|c|c|c|c|c|c|}
\hline \multirow{2}{*}{ Bitumen type } & \multirow{2}{*}{ Confidence level } & \multicolumn{2}{|c|}{$95 \%$} & \multicolumn{2}{|c|}{$90 \%$} & \multicolumn{2}{|c|}{$85 \%$} \\
\hline & & Lower & Upper & Lower & Upper & Lower & Upper \\
\hline \multirow{5}{*}{ U15 } & $\delta$ & 10.09 & 10.32 & 10.12 & 10.28 & 10.13 & 10.27 \\
\hline & $\alpha$ & -11.37 & -10.59 & -11.24 & -10.72 & -11.21 & -10.75 \\
\hline & $\lambda$ & 3.52 & 3.66 & 3.55 & 3.64 & 3.55 & 3.63 \\
\hline & $\beta$ & 2.18 & 2.46 & 2.23 & 2.41 & 2.24 & 2.40 \\
\hline & $\gamma$ & 0.57 & 0.58 & 0.57 & 0.57 & 0.57 & 0.57 \\
\hline \multirow{5}{*}{ U70 } & $\delta$ & -7.06 & -5.33 & -6.92 & -5.47 & -6.83 & -5.56 \\
\hline & $\alpha$ & 13.06 & 14.38 & 13.17 & 14.27 & 13.24 & 14.20 \\
\hline & $\lambda$ & 2.57 & 4.37 & 2.71 & 4.23 & 2.81 & 4.13 \\
\hline & $\beta$ & 0.00 & 0.14 & 0.01 & 0.12 & 0.01 & 0.12 \\
\hline & $\gamma$ & -0.56 & -0.42 & -0.55 & -0.43 & -0.54 & -0.44 \\
\hline \multirow{5}{*}{ SBS } & $\delta$ & 0.96 & 1.15 & 0.98 & 1.13 & 0.99 & 1.12 \\
\hline & $\alpha$ & 6.16 & 6.56 & 6.19 & 6.53 & 6.21 & 6.51 \\
\hline & $\lambda$ & 0.62 & 0.69 & 0.63 & 0.69 & 0.63 & 0.68 \\
\hline & $\beta$ & 0.15 & 0.20 & 0.15 & 0.20 & 0.16 & 0.20 \\
\hline & $\gamma$ & -0.42 & -0.38 & -0.41 & -0.38 & -0.41 & -0.38 \\
\hline
\end{tabular}

increased, the confidence interval of model parameters correspondingly grew. However, in the CAM and CAM $\left(G_{g}\right)$ models, MCB width change with the increase of confidence level was not significant. It illustrated that although the confidence level was altered, the constructed master-curve bands were not sensitive to the change of confidence level. In this condition, given that a modeling error existed, the utilization of the CAM and CAM $\left(G_{g}\right)$ models would not significantly change the final constructed master curve. This modeling stability, to some extent, can alleviate the modeling uncertainty of the master curve.

Nevertheless, this finding brings about some other issues when comparing master curves between similar bitumen types. Assuming that bitumen was aged insignificantly, the 


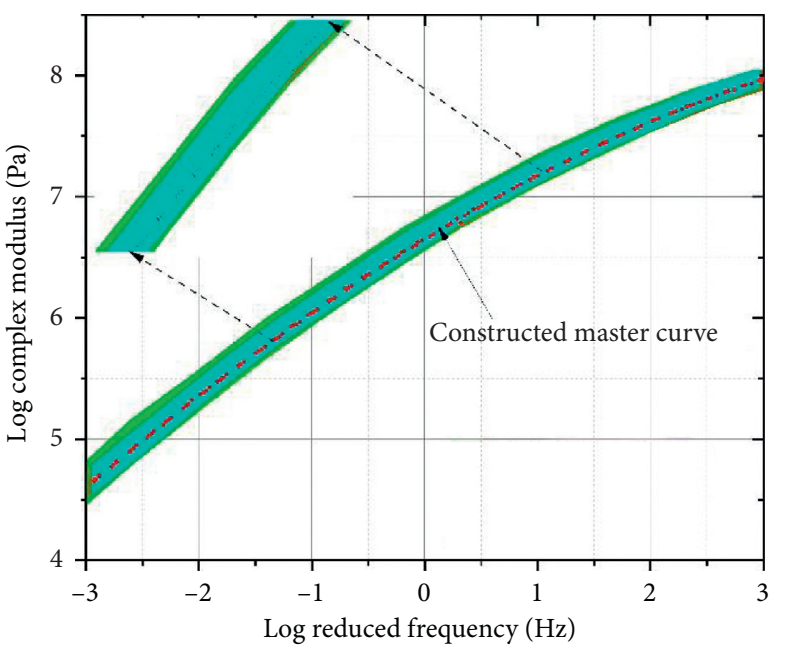

U15_CAM_95\%

U15_CAM $90 \%$

U15_CAM_85\%

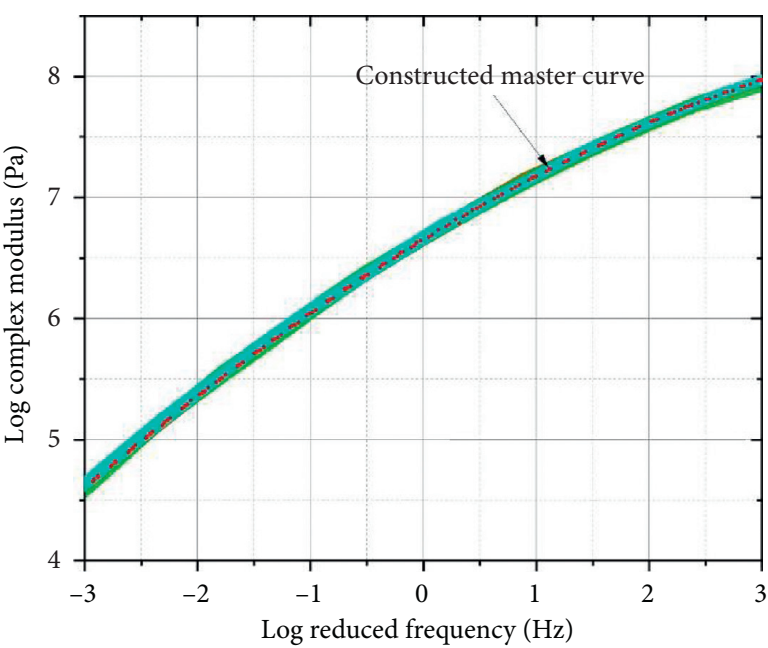

U15_CAM (Gg)_95\%

U15_CAM (Gg)_90\%

U15_CAM (Gg)_85\%

(b)

(a)

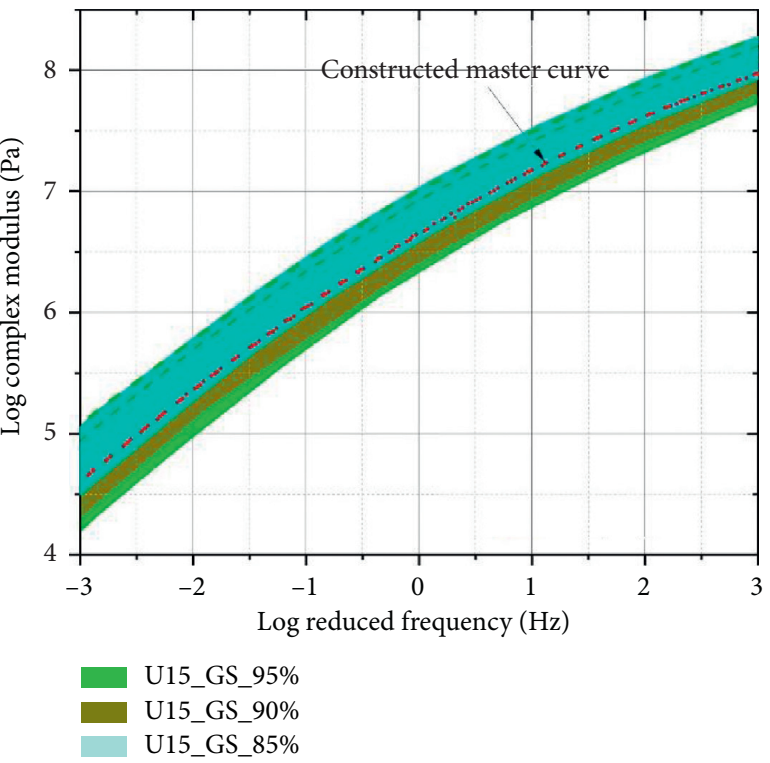

(c)

FIGURE 7: Master-curve bands constructed with different confidence levels. (a) CAM model. (b) CAM (Gg) model. (c) Generalized sigmoidal model.

master curve of aged bitumen was similar to its origin bitumen. In this condition, the master-curve comparison cannot reveal the essential variation caused by aging, considering the modeling uncertainty.

3.4. Effect of Bitumen Aging. Figure 8 compares the mastercurve bands of unaged and aged bitumen (U15 and A15) using different rheological models. When the rheological model was selected as the generalized sigmoidal model, the master-curve band of A15 overlapped with U15, especially at high frequencies. The master-curve band refers to all possible constructed master curves if modeling uncertainty existed. Therefore, the overlapped area claimed the possibility that the master curves of A15 and U15 were close to each other. In this case, the effect of aging on rheological properties of bitumen might be enervated. Therefore, it is not appropriate for researchers to identify the aging effect using a generalized sigmoidal model to model uncertainty.

As can be seen from Figures 8(b) and 8(c), the overlapping area of master-curve bands for A15 and U15 can be considerably reduced using the CAM or CAM $\left(G_{g}\right)$ models. It can be found that the overlapping area was relevant to the master-curve band's width. As aforementioned, bitumen showed the smallest sensitivity to the CAM $\left(G_{g}\right)$ model. Therefore, the width of the master-curve band tended to 


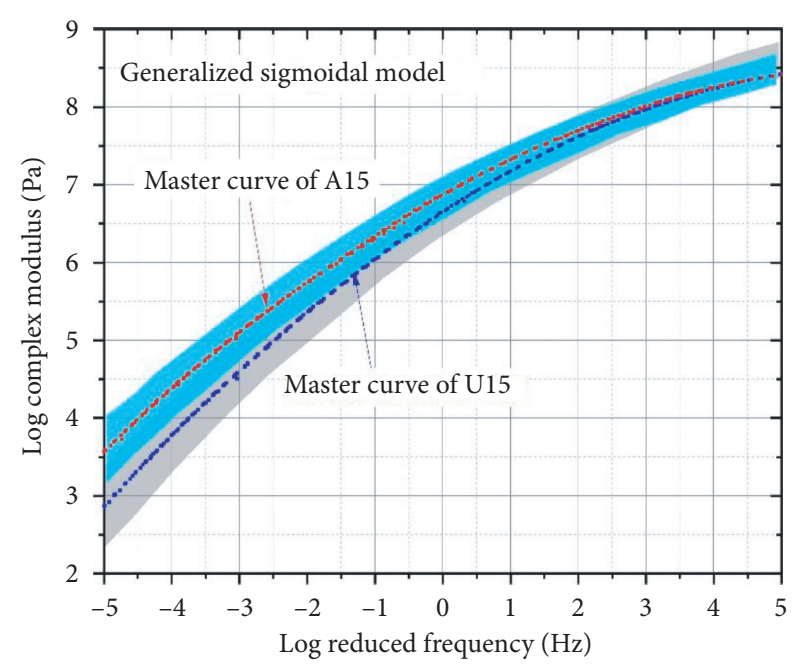

(a)

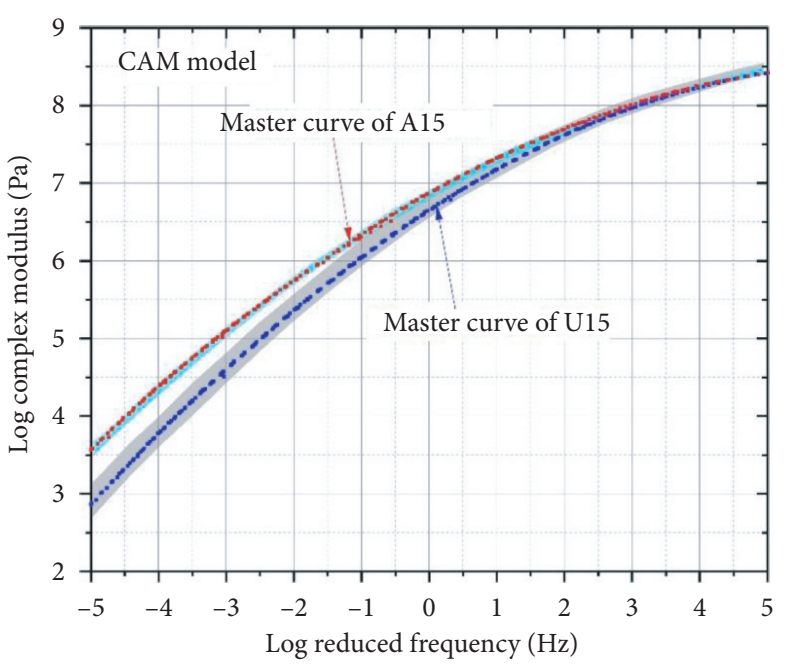

(b)

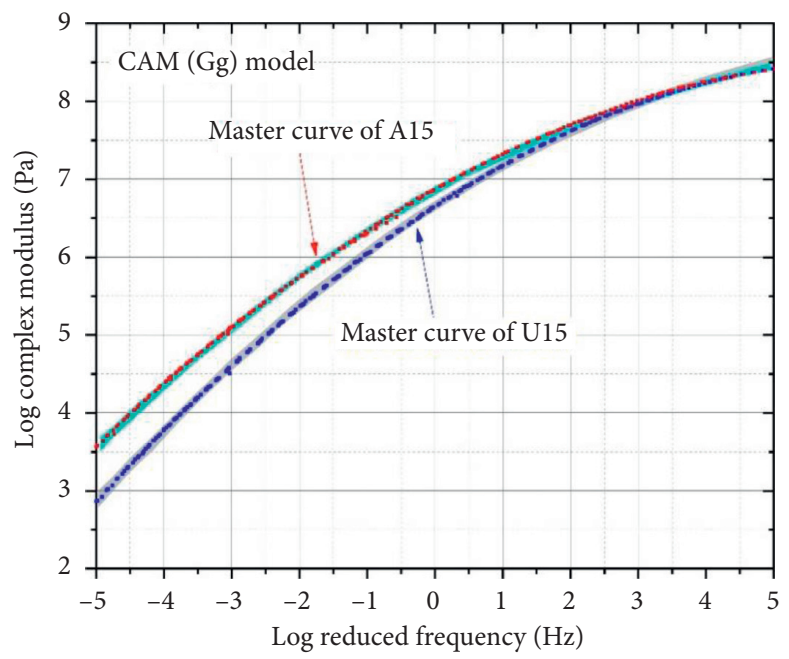

(c)

Figure 8: Master-curve band comparison between unaged and aged bitumen. (a) Generalized sigmoidal model. (b) CAM model. (c) CAM (Gg) model.

present in a small value. A small value of band width indicated a small deviation caused by the modeling uncertainty. Hence, it can be concluded that the modeling uncertainty can be eliminated as much as possible if an appropriate rheological model is used. In previous studies of rheological modeling of bitumen binders, only the goodness of fit was concerned. The introduction of master-curve bands revealed that the rheological model's selection should consider satisfying fitting results and the resistance ability to the modeling uncertainty.

\section{Conclusion and Outlook}

4.1. Conclusion. In the engineering field, measurement and modeling uncertainty represent a critical topic. However, there is a lack of studies considering the uncertainty in the construction of the master curve. For this reason, this study proposed a method to conclude the uncertainty property. The fundamental technology includes two parts, which are separately relative to the measurement error and modeling uncertainty. For the measurement error, the coefficient of variation was used to describe the error quantitatively. The normal distribution fitting was adopted to obtain the parameter intervals at a specific confidence level for the modeling uncertainty. Consequently, the master-curve band can be generated through a calculating experiment via MATLAB. Through investigating the fundamental properties of the master-curve band, some critical findings were concluded as follows:

(1) Ignorance of modeling uncertainty in the estimation of aging or modification would possibly hinder an appropriate conclusion to be achieved, especially when the aging or modification degree is relatively small.

(2) Master-curve bands constructed for the same bitumen were different on account of various rheological models. The width of the master band is dominated 
by the sensitivity of bitumen to the rheological model.

(3) The sensitivity of bitumen to rheological models was determined by two factors. The first is the number of active parameters in the selected rheological model. The second is related to the confidence interval of active parameters.

(4) In this study, the generalized sigmoidal model showed the widest master-curve band, while the CAM $\left(G_{g}\right)$ model performed the best. Therefore, it is recommended to use the CAM $\left(G_{g}\right)$ model rather than the generalized sigmoidal model to analyze the aging effect.

4.2. Outlook. It is undeniable that this proposed method could not be an efficient approach to characterize the master-curve construction's uncertainty information. This study's primary purpose is to introduce uncertainty into the master curve to strengthen the reliability of the master curve. It is believed that much work should be done to modify the master-curve band toward an efficient and thoughtful approach to include the uncertainty information in the master curve. From the evaluation of the authors, the master-curve band can be modified from the following perspectives:

(1) Notably, a detailed standard procedure to construct the master-curve band was not recommended. The reason is that limited types of bitumen and rheological models were investigated in this study. Therefore, an investigation with more samples and models is expected to validate some arguments in constructing the master-curve band. In addition, more potential influence factors of the master-curve band will need to be discussed.

(2) The coefficient of variation used in this study was based on the RILEM research. However, it will be more rational when the coefficient of variation is determined by repeatedly conducting the DSR measurements.

(3) Normal distribution fitting was adopted to feature the modeling uncertainty, and all the parameters were assumed to be independent of each other. However, the data space of model parameters can be established by other advanced uncertainty analysis methods.

\section{Data Availability}

The data used to support the findings of this study are included within the article.

\section{Conflicts of Interest}

The authors declare that they have no conflicts of interest.

\section{Acknowledgments}

The authors would like to acknowledge the National Natural Science Foundation of China (no. 52078190) and the
German Research Foundation (O.E. 514/10-1). The authors also gratefully acknowledge financial support from the China Scholarship Council (CSC no. 201706710009).

\section{References}

[1] Y. Gao, Y. Zhang, F. Gu, T. Xu, and H. Wang, "Impact of minerals and water on bitumen-mineral adhesion and debonding behaviours using molecular dynamics simulations," Construction and Building Materials, vol. 1711016 pages, 2018.

[2] Y. Gao, Y. Zhang, Y. Yang, J. Zhang, and F. Gu, "Molecular dynamics investigation of interfacial adhesion between oxidised bitumen and mineral surfaces," Applied Surface Science, vol. 4791292 pages, 2019.

[3] S. Wu, Q. Liu, J. Yang, R. Yang, and J. Zhu, "Study of adhesion between crack sealant and pavement combining surface free energy measurement with molecular dynamics simulation," Construction and Building Materials, vol. 240, Article ID 117900, 2020.

[4] Y. Gao, L. Li, and Y. Zhang, "Modeling crack propagation in bituminous binders under a rotational shear fatigue load using pseudo $j$-integral paris' law," Transportation Research Record: Journal of the Transportation Research Board, vol. 2674, no. 1, pp. 94-103, 2020.

[5] Z. Zhang, Q. Liu, Qi Wu, H. Xu, P. Liu, and M. Oeser, "Damage evolution of asphalt mixture under freeze-thaw cyclic loading from a mechanical perspective," International Journal of Fatigue, vol. 142, Article ID 105923, 2021.

[6] E. Masad, C.-W. Huang, G. Airey, and A. Muliana, "Nonlinear viscoelastic analysis of unaged and aged asphalt binders," Construction and Building Materials, vol. 22, no. 11, pp. 2170-2179, 2008.

[7] Q. Liu, J. Wu, X. Qu, C. Wang, and M. Oeser, "Investigation of bitumen rheological properties measured at different rheometer gap sizes," Construction and Building Materials, vol. 265, Article ID 120287, 2020.

[8] G. D. Airey, "Use of black diagrams to identify inconsistencies in rheological data," Road Materials and Pavement Design, vol. 3, no. 4, pp. 403-424, 2002.

[9] D. I. Alhamali, J. Wu, Q. Liu et al., "Physical and rheological characteristics of polymer modified bitumen with nanosilica particles," Arabian Journal for Science and Engineering, vol. 41, no. 4, pp. 1521-1530, 2016.

[10] P. Ahmedzade, A. Fainleib, T. Günay, and O. Grygoryeva, "Modification of bitumen by electron beam irradiated recycled low density polyethylene," Construction and Building Materials, vol. 69, pp. 1-9, 2014.

[11] G. King, M. Anderson, D. Hanson, and P. Blankenship, "Using black space diagrams to predict age-induced cracking," in Proceedings of the 7th RILEM International Conference on Cracking in Pavements, Dordrecht, Netherlands, August 2012.

[12] Benedetto, Herve Di, F. Olard, C. . Sauzéat, and B. Delaporte, "Linear viscoelastic behaviour of bituminous materials: from binders to mixes," Road Materials and Pavement Design, vol. 5, no. 1, pp. 163-202, 2004.

[13] S. Mangiafico, H. Di Benedetto, C. Sauzéat, F. Olard, S. Pouget, and L. Planque, "New method to obtain viscoelastic properties of bitumen blends from pure and reclaimed asphalt pavement binder constituents," Road Materials and Pavement Design, vol. 15, no. 2, pp. 312-329, 2014.

[14] H. M. Nguyen, S. Pouget, H. di Benedetto, and C. Sauzéat, "Time-temperature superposition principle for bituminous 
mixtures," Revue européenne de génie civil, vol. 13, no. 9, pp. 1095-1107, 2009.

[15] N. I. Nur, E. Chailleux, and G. D. Airey, "A comparative study of the influence of shift factor equations on master curve construction," International Journal of Pavement Research and Technology, vol. 4, no. 6, pp. 324-336, 2011.

[16] F. P. Germann and R. L. Lytton, "Methodology for predjcting the reflection cracking life of asphalt concrete overlays," Texas A\&M Transportation Institute, Austin, TX, USA, Res. Rpt. No. 207-5, 1979.

[17] L. Francken and C. Clauwaert, "Characterization and structural assessment of bound materials for flexible road structures," in Proceedings of the 6 International Conference on Asphalt Pavements, pp. 130-144, Ann Arbor, MS, USA, August 1987.

[18] M. Williams and D. Ferry, "The temperature dependence of relaxation mechanisms in amorphous polymers and other glass-forming liquids," Journal of the American Chemical Society, vol. 77, no. 14, pp. 3701-3707, 1955.

[19] T. K. Pellinen, M. W. Witczak, and R. F. Bonaquist, "Asphalt mix master curve construction using sigmoidal fitting function with non-linear least squares optimization," Geotechnical Special Publication, vol. 2576 pages, 2004.

[20] M. Yusoff Nur Izzi, M.-S. Ginoux, G. Dan Airey, and M. Rosli Hainin, "Modelling the linear viscoelastic rheological properties of base bitumens," Malaysian Journal of Civil Engineering, vol. 22, no. 1, 2010.

[21] E. Chailleux, R. Guy, C. Such, and C. De La Roche, "A mathematical-based master-curve construction method applied to complex modulus of bituminous materials," Road Materials and Pavement Design, vol. 7, no. 1, 2006.

[22] M. Yusoff Nur Izzi, M. T. Shaw, and G. D. Airey, "Modelling the linear viscoelastic rheological properties of bituminous binders," Construction and Building Materials, vol. 25, no. 5, pp. 2171-2189, 2011.

[23] J. Carswell, M. J. Claxton, and P. J. Green, "Dynamic shear rheometers: making accurate measurements on bitumens," in Proceedings of Conference of the Australian Road Research Board, pp. 219-237, Canberra. Australia, August 1998.

[24] J. Wu, M. Y. Nur Izzi, F. Mohd Jakarni, and M. Rosli Hainin, "Correction of compliance errors in the dynamic shear modulus of bituminous binders data," Sains Malaysiana, vol. 42, no. 6, pp. 783-792, 2013.

[25] M. N. Partl and H. Bahia, Advances in Interlaboratory Testing and Evaluation of Bituminous Materials: State-Of-The-Art Report of the RILEM Technical Committee 206-ATB, Springer Science \& Business Media, Berlin, Germany, 2012.

[26] N. I. Nur, D. Mounier, G. Marc-Stéphane, M. Rosli Hainin, G. D. Airey, and Hervé Di Benedetto, "Modelling the rheological properties of bituminous binders using the 2S2P1D model," Construction and Building Materials, vol. 38, pp. 395-406, 2013.

[27] R. de Carteret, C. Lee, J. Metcalf, and J. Rebbechi, Guide to Pavement Technology: Part 8: Pavement Construction Indian, Academy of Sciences, Bengaluru, Karnataka, India, 2009.

[28] S. M. Asgharzadeh, N. Tabatabaee, K. Naderi, M. Partl, and M. Partl, "An empirical model for modified bituminous binder master curves," Materials and Structures, vol. 46, no. 9, pp. 1459-1471, 2013.

[29] D. Wang, Q. Liu, Q. Yang, C. Tovar, Y. Tan, and M. Oeser, "Thermal oxidative and ultraviolet ageing behaviour of nanomontmorillonite modified bitumen," Road Materials and Pavement Design, vol. 22, no. 1, pp. 121-139, 2019.
[30] T. O. Medani, M. Tech, and M. Huurman, "Constructing the stiffness master curves for asphaltic mixes," Civil Engineering, vol. 25, no. 12, pp. 1813-1821, 2003.

[31] N. Yusoff Nur Izzi, F. M. Jakarni, V. H. Nguyen, M. R. Hainin, and G. D. Airey, "Modelling the rheological properties of bituminous binders using mathematical equations," Construction and Building Materials, vol. 40, pp. 174-188, 2013. 